\title{
SHARP POWER MEAN BOUNDS FOR THE SECOND NEUMAN MEAN
}

\author{
XIAO-HONG HE, YUE-YING YANG, AND WEI-MAO QIAN
}

Received 20 November, 2016

\begin{abstract}
In this paper, we prove that the double inequality $M_{\alpha}(a, b)<N_{G Q}(a, b)<M_{\beta}(a, b)$ holds for all $a, b>0$ with $a \neq b$ if and only if $\alpha \leq 2 \log 2 /(5 \log 2-2 \log \pi)=1.1785 \cdots$ and $\beta \geq 4 / 3$, where $N_{G Q}(a, b)=\left[G(a, b)+Q^{2}(a, b) / U(a, b)\right] / 2$ is the second Neuman mean, $G(a, b)=\sqrt{a b}, Q(a, b)=\sqrt{\left(a^{2}+b^{2}\right) / 2}$ and $U(a, b)=(a-b) /\left[\sqrt{2} \tan ^{-1}((a-b) / \sqrt{2 a b})\right]$ are the geometric, quadratic and Yang mean of $a$ and $b$, respectively.
\end{abstract}

2010 Mathematics Subject Classification: 26E60

Keywords: Schwab-Borchardt mean, second Neuman mean, power mean, geometric mean, Yang mean

\section{INTRODUCTION}

For $p \in \mathbb{R}$ and $a, b>0$ with $a \neq b$, the $p$ th power mean $M_{p}(a, b)[14]$ of $a$ and $b$ is defined by

$$
M_{p}(a, b)= \begin{cases}\left(\frac{a^{p}+b^{p}}{2}\right)^{1 / p} & \text { if } p \neq 0 \\ \sqrt{a b} & \text { if } p=0 .\end{cases}
$$

It is well known that the power mean $M_{p}(a, b)$ is continuous and strictly increasing with respect to $p \in \mathbb{R}$ for fixed $a, b>0$ with $a \neq b$. Many bivariate means are the special cases of the power mean, for example, $M_{0}(a, b)=G(a, b)=\sqrt{a b}$, $M_{1}(a, b)=A(a, b)=(a+b) / 2$ and $M_{2}(a, b)=Q(a, b)=\sqrt{\left(a^{2}+b^{2}\right) / 2}$ are respectively the arithmetic, geometric and quadratic means. Many properties for the power mean can be found in the literature[2-5, 11, 22, 24, 26, 31,36].

The Schwab-Borchardt mean $S B(a, b)[16,17]$ defined by

$$
S B(a, b)= \begin{cases}\frac{\sqrt{b^{2}-a^{2}}}{\cos ^{-1}(a / b)} & \text { if } a<b \\ \frac{\sqrt{a^{2}-b^{2}}}{\cosh ^{-1}(a / b)} & \text { if } a>b,\end{cases}
$$

where $\cos ^{-1}(x)$ and $\cosh ^{-1}(x)=\log \left(x+\sqrt{x^{2}-1}\right)$ are the inverse cosine and inverse hyperbolic cosine functions, respectively.It is well-known that $S B(a, b)$ is strictly 
increasing in both $a$ and $b$, nonsymmetric and homogeneous of degree 1 with respect to $a$ and $b$. Many symmetric bivariate means are special cases of the SchwabBorchardt mean, for example, the first Seiffert mean $P(a, b)$, second Seiffert mean $T(a, b)$, Neuman-Sándor mean $M(a, b)$, logarithmic mean $L(a, b)$ and Yang mean $U(a, b)[29]$ are respectively defined by

and

$$
\begin{gathered}
P(a, b)=\frac{a-b}{2 \sin ^{-1}[(a-b) /(a+b)]}=S B[G(a, b), A(a, b)], \\
T(a, b)=\frac{a-b}{2 \tan ^{-1}[(a-b) /(a+b)]}=S B[A(a, b), Q(a, b)], \\
M(a, b)=\frac{a-b}{2 \sinh ^{-1}[(a-b) /(a+b)]}=S B[Q(a, b), A(a, b)], \\
L(a, b)=\frac{a-b}{2 \tanh ^{-1}[(a-b) /(a+b)]}=S B[A(a, b), G(a, b)],
\end{gathered}
$$

$$
U(a, b)=\frac{a-b}{\sqrt{2} \tan ^{-1}[(a-b) / \sqrt{2 a b}]}=S B[G(a, b), Q(a, b)] .
$$

In 2014, Neuman [15] found a new bivariate means derived from the SchwabBorchardt mean

$$
N(a, b)=\frac{1}{2}\left[a+\frac{b^{2}}{S B(a, b)}\right] .
$$

We call $N(a, b)$ is the second Neuman mean[19]. Let $a>b, v=(a-b) /(a+b) \in$ $(0,1)$, then Neuman [15] gave explicit formulas

$$
\begin{aligned}
& N_{A G}(a, b)=\frac{1}{2} A(a, b)\left[1+\left(1-v^{2}\right) \frac{\tanh ^{-1}(v)}{v}\right], N_{G A}(a, b)=\frac{1}{2} A(a, b)\left[\sqrt{1-v^{2}}+\frac{\sin ^{-1}(v)}{v}\right], \\
& N_{Q A}(a, b)=\frac{1}{2} A(a, b)\left[\sqrt{1+v^{2}}+\frac{\sinh ^{-1}(v)}{v}\right], N_{A Q}(a, b)=\frac{1}{2} A(a, b)\left[1+\left(1+v^{2}\right) \frac{\tan ^{-1}(v)}{v}\right] .
\end{aligned}
$$

and proved that the inequalities

$G(a, b)<N_{A G}(a, b)<N_{G A}(a, b)<A(a, b)<N_{Q A}(a, b)<N_{A Q}(a, b)<Q(a, b)$ for $a, b>0$ with $a \neq b$.

Very recently, Shen et. al. [21] found a new mean $N_{G Q}(a, b)$ derived from the Schwab- Borchardt mean. Let $a>b, u=(a-b) / \sqrt{2 a b} \in(0,+\infty)$, then explicit formulas for $N_{G Q}(a, b)$ be in the following:

$$
N_{G Q}(a, b)=\frac{1}{2} G(a, b)\left[1+\left(1+u^{2}\right) \frac{\tan ^{-1}(u)}{u}\right] .
$$

Recently, the bounds involving the power and the Schwab-Borchardt means has been the subject of intensive research. In particular, many remarkable inequalities for the power mean, Schwab-Borchardt mean and their related means can be found in the literature $[1,6-10,12,13,18-21,23,25,27-30,32-35]$. 
Radó[20] (see also [13, 18,23]) proved that the double inequalities

$$
M_{p}(a, b)<L(a, b)<M_{q}(a, b), M_{\lambda}(a, b)<I(a, b)<M_{\mu}(a, b)
$$

hold for all $a, b>0$ with $a \neq b$ if and only if $p \leq 0, q \geq 1 / 3, \lambda \leq 2 / 3$ and $\mu \geq \log 2$, where $I(a, b)=\left(a^{a} / b^{b}\right)^{1 /(a-b)} / e$ is the indentric mean of $a$ and $b$.

In $[7-10,12,28]$, the authors proved that $p_{1}=\log 2 / \log \pi, q_{1}=2 / 3, p_{2}=$ $\log 2 /(\log \pi-\log 2), q_{2}=5 / 3, p_{3}=\log 2 / \log [2 \log (1+\sqrt{2})]$ and $q_{3}=4 / 3$ are the best possible parameters such that the double inequalities

$$
\begin{aligned}
& M_{p_{1}}(a, b)<P(a, b)<M_{q_{1}}(a, b), \\
& M_{p_{2}}(a, b)<T(a, b)<M_{q_{2}}(a, b), \\
& M_{p_{3}}(a, b)<M(a, b)<M_{q_{3}}(a, b)
\end{aligned}
$$

hold for all $a, b>0$ with $a \neq b$.

Chu [6] and Yang [30] proved that the double inequalities

$$
M_{\lambda_{1}}(a, b)<X(a, b)<M_{\mu_{1}}(a, b), M_{\lambda_{2}}(a, b)<U(a, b)<M_{\mu_{2}}(a, b)
$$

hold for all $a, b>0$ with $a \neq b$ if and only if $\lambda_{1} \leq 1 / 3, \mu_{1} \geq \log 2 /(1+\log 2)$, $\lambda_{2} \leq 2 \log 2 /(2 \log \pi-\log 2)$ and $\mu_{2} \geq 4 / 3$, where $X(a, b)=A e^{G / P-1}$ is the Sándor mean of $a$ and $b$.

In [21], the authors proved the double inequalities

$$
\begin{gathered}
\alpha_{1} Q(a, b)+\left(1-\alpha_{1}\right) G(a, b)<N_{G Q}(a, b)<\beta_{1} Q(a, b)+\left(1-\beta_{1}\right) G(a, b), \\
\frac{\alpha_{2}}{G(a, b)}+\frac{1-\alpha_{2}}{Q(a, b)}<\frac{1}{N_{G Q}(a, b)}<\frac{\beta_{2}}{G(a, b)}+\frac{1-\beta_{2}}{Q(a, b)}, \\
\alpha_{3} Q(a, b)+\left(1-\alpha_{3}\right) U(a, b)<N_{G Q}(a, b)<\beta_{3} Q(a, b)+\left(1-\beta_{3}\right) U(a, b)
\end{gathered}
$$

hold for all $a, b>0$ with $a \neq b$ if and only if $\alpha_{1} \leq 2 / 3, \beta_{1} \geq \pi / 4, \alpha_{2} \leq 0, \beta_{2} \geq 1 / 3$, $\alpha_{3} \leq 0$ and $\beta_{3} \geq\left(\pi^{2}-8\right) /[4(\pi-2)]=0.4094 \cdots$

The main purpose of this paper is to present the best possible parameter $\alpha$ and $\beta$ such that the double inequalities $M_{\alpha}(a, b)<N_{G Q}(a, b)<M_{\beta}(a, b)$ hold for all $a, b>0$ with $a \neq b$.

\section{MAIN RESULT}

In order to prove our main result we need a lemma, which we present in this section.

Lemma 1. Let $p \in \mathbb{R}$, and

$$
\begin{array}{r}
f(x)=x^{2 p+2}+x^{2 p+1}+5 x^{2 p}+x^{2 p-1}+(2 p-3) x^{p+3}-4 x^{p+2}+4 x^{p} \\
-(2 p-3) x^{p-1}-x^{3}-5 x^{2}-x-1
\end{array}
$$

Then the following statements are true:

(1) If $p=4 / 3$, then $f(x)>0$ for all $x \in(1,+\infty)$; 
(2) If $p=2 \log 2 /(5 \log 2-2 \log \pi)=1.1785 \cdots$, then there exists $\lambda \in(1,+\infty)$ such that $f(x)<0$ for $x \in(1, \lambda)$ and $f(x)>0$ for $x \in(\lambda,+\infty)$.

Proof. For part (1), if $p=4 / 3$, then (2.1) becomes

$$
\begin{aligned}
f(x)=\frac{1}{3}\left(x^{2 / 3}-1\right)^{3}\left(3 x^{8 / 3}-x^{7 / 3}+9 x^{2}+6 x^{4 / 3}+9 x^{2 / 3}-x^{1 / 3}+3\right) \\
=\frac{1}{3}\left(x^{2 / 3}-1\right)^{3}\left[2 x^{8 / 3}+x^{7 / 3}\left(x^{1 / 3}-1\right)+9 x^{2}+6 x^{4 / 3}\right. \\
\left.+8 x^{2 / 3}+x^{1 / 3}\left(x^{1 / 3}-1\right)+3\right] \\
>\frac{1}{3}\left(x^{2 / 3}-1\right)^{3}\left(2 x^{8 / 3}+9 x^{2}+6 x^{4 / 3}+8 x^{2 / 3}+3\right)
\end{aligned}
$$

for $x \in(1,+\infty)$.

Therefore, part (1) follows from (2.2).

For part (2), let $p=2 \log 2 /(5 \log 2-2 \log \pi)=1.1785 \cdots, f_{1}(x)=f^{\prime}(x), f_{2}(x)=$ $f_{1}^{\prime}(x), f_{3}(x)=f_{2}^{\prime}(x), f_{4}(x)=x^{5-p} f_{3}^{\prime}(x)$. Then elaborated computations lead to

$$
\lim _{x \rightarrow 1} f(x)=0, \lim _{x \rightarrow+\infty} f(x)=+\infty
$$

$$
\begin{gathered}
f_{1}(x)=2(p+1) x^{2 p+1}+(2 p+1) x^{2 p}+10 p x^{2 p-1}+(2 p-1) x^{2 p-2} \\
+(p+3)(2 p-3) x^{p+2}-4(p+2) x^{p+1}+4 p x^{p-1} \\
-(p-1)(2 p-3) x^{p-2}-3 x^{2}-10 x-1 \\
\lim _{x \rightarrow 1} f_{1}(x)=24\left(p-\frac{4}{3}\right)<0, \lim _{x \rightarrow+\infty} f_{1}(x)=+\infty
\end{gathered}
$$

$$
\begin{gathered}
f_{2}(x)=2(p+1)(2 p+1) x^{2 p}+2 p(2 p+1) x^{2 p-1}+10 p(2 p-1) x^{2 p-2} \\
+2(p-1)(2 p-1) x^{2 p-3}+(p+2)(p+3)(2 p-3) x^{p+1} \\
-4(p+1)(p+2) x^{p}+4 p(p-1) x^{p-2} \\
-(p-1)(p-2)(2 p-3) x^{p-3}-6 x-10 \\
\lim _{x \rightarrow 1} f_{2}(x)=24(2 p+1)\left(p-\frac{4}{3}\right)<0, \lim _{x \rightarrow+\infty} f_{2}(x)=+\infty
\end{gathered}
$$

$$
\begin{gathered}
f_{3}(x)=4 p(p+1)(2 p+1) x^{2 p-1}+2 p\left(4 p^{2}-1\right) x^{2 p-2}+20 p(p-1)(2 p-1) x^{2 p-3} \\
+2(p-1)(2 p-1)(2 p-3) x^{2 p-4}+(p+1)(p+2)(p+3)(2 p-3) x^{p} \\
-4 p(p+1)(p+2) x^{p-1}+4 p(p-1)(p-2) x^{p-3} \\
-(p-1)(p-2)(p-3)(2 p-3) x^{p-4}-6 \\
\lim _{x \rightarrow 1} f_{3}(x)=4\left(22 p^{3}-33 p^{2}+17 p-12\right)<0, \lim _{x \rightarrow+\infty} f_{3}(x)=+\infty
\end{gathered}
$$




$$
\begin{aligned}
& f_{4}(x)=4 p(p+1)\left(4 p^{2}-1\right) x^{p+3}+4 p(p-1)\left(4 p^{2}-1\right) x^{p+2} \\
& +20 p(p-1)(2 p-1)(2 p-3) x^{p+1}+4(p-1)(p-2)(2 p-1)(2 p-3) x^{p} \\
& \quad+p(p+1)(p+2)(p+3)(2 p-3) x^{4}-4 p\left(p^{2}-1\right)(p+2) x^{3} \\
& +4 p(p-1)(p-2)(p-3) x-(p-1)(p-2)(p-3)(p-4)(2 p-3) \\
& =a_{0} x^{p+3}+a_{2} x^{p+2}+a_{4} x^{p+1}+a_{5} x^{p}+a_{1} x^{4}+a_{3} x^{3}+a_{6} x+a_{7} .
\end{aligned}
$$

Note that

$$
\begin{gathered}
p+3>4>p+2>3>p+1>p>1>0, \\
a_{0}>0, a_{1}<0, a_{2}>0, a_{3}<0, a_{4}<0, a_{5}>0, a_{6}>0, a_{7}<0, \\
23 p^{2}-43 p+12=-6.7311 \cdots<0,2 p^{3}-37 p^{2}+89 p-48=8.7726 \cdots>0, \\
2 p^{3}+119 p^{2}-125 p+70=91.2430 \cdots>0, \\
a_{0}+a_{1}=p\left(p^{2}-1\right)\left(2 p^{2}+25 p+22\right)>0, \\
a_{2}+a_{3}+a_{4}=4 p(p-1)\left(23 p^{2}-43 p+12\right) \\
a_{5}+a_{6}+a_{7}=(p-1)(2-p)\left(2 p^{3}-37 p^{2}+89 p-48\right), \\
\sum_{i=0}^{4} a_{i}=p(p-1)\left(2 p^{3}+119 p^{2}-125 p+70\right),
\end{gathered}
$$

It follows from (2.7)-(2.15) that

$$
\begin{aligned}
f_{4}(x)>\left(a_{0}+a_{1}\right) x^{4}+\left(a_{2}+a_{3}+a_{4}\right) x^{3} & +\left(a_{5}+a_{6}+a_{7}\right) x \\
& >\sum_{i=0}^{4} a_{i} x^{4}+\left(a_{5}+a_{6}+a_{7}\right) x>0
\end{aligned}
$$

for $x \in(1,+\infty)$.

From (2.16) we clearly see that $f_{3}(x)$ is strictly increasing on $(1,+\infty)$. Then (2.6) leads to the conclusion that there exists $\lambda_{1}>1$ such that $f_{2}(x)$ is strictly decreasing on $\left(1, \lambda_{1}\right]$ and strictly increasing on $\left[\lambda_{1},+\infty\right)$.

It follows from (2.5) and the piecewise monotonicity of $f_{2}(x)$, we conclude that there exists $\lambda_{2} \in(1,+\infty)$ such that $f_{1}(x)$ is strictly decreasing on $\left(1, \lambda_{2}\right]$ and strictly increasing on $\left[\lambda_{2},+\infty\right)$.

From (2.4) and the piecewise monotonicity of $f_{1}(x)$ that there exists $\lambda_{3} \in(1,+\infty)$ such that $f(x)$ is strictly decreasing on $\left(1, \lambda_{3}\right]$ and strictly increasing on $\left[\lambda_{3},+\infty\right)$.

Therefore, part (2) follows from (2.3) and the piecewise monotonicity of $f(x)$.

Theorem 1. The double inequality

$$
M_{\alpha}(a, b)<N_{G Q}(a, b)<M_{\beta}(a, b),
$$

holds for all $a, b>0$ with $a \neq b$ if and only if $\alpha \leq 2 \log 2 /(5 \log 2-2 \log \pi)=$ $1.1785 \cdots$ and $\beta \geq 4 / 3$. 
Proof. Since $N_{G Q}(a, b)$ and $M_{p}(a, b)$ are symmetric and homogenous of degree 1 , we assume that $a>b>0$. Let $x=a / b \in(1,+\infty), p \in \mathbb{R}_{+}$. Then we have

$$
\begin{aligned}
& \log \left[N_{G Q}(a, b)\right]-\log \left[M_{p}(a, b)\right] \\
= & \log \left[2 \sqrt{x}(x-1)+\sqrt{2}\left(x^{2}+1\right) \tan ^{-1}\left(\frac{x-1}{\sqrt{2 x}}\right)\right]-\log [4(x-1)]-\frac{1}{p} \log \left(\frac{x^{p}+1}{2}\right) .
\end{aligned}
$$

Let

$$
\begin{aligned}
F(x)= & \log \left[2 \sqrt{x}(x-1)+\sqrt{2}\left(x^{2}+1\right) \tan ^{-1}\left(\frac{x-1}{\sqrt{2 x}}\right)\right] \\
& -\log [4(x-1)]-\frac{1}{p} \log \left(\frac{x^{p}+1}{2}\right)
\end{aligned}
$$

Then simple computations lead to

$$
\begin{gathered}
\lim _{x \rightarrow 1^{+}} F(x)=0, \\
\lim _{x \rightarrow+\infty} F(x)=\frac{1}{p} \log 2+\log \pi-5 \log \sqrt{2}, \\
F^{\prime}(x)=\frac{x^{p+1}+2 x^{p}-x^{p-1}-x^{2}+2 x+1}{(x-1)\left(x^{p}+1\right)\left[2 \sqrt{x}(x-1)+\sqrt{2}\left(x^{2}+1\right) \tan ^{-1}\left(\frac{x-1}{\sqrt{2 x}}\right)\right]} F_{1}(x),
\end{gathered}
$$

where

$$
\begin{gathered}
F_{1}(x)=\frac{2 \sqrt{x}(x-1)\left(x^{p-1}+1\right)}{x^{p+1}+2 x^{p}-x^{p-1}-x^{2}+2 x+1}-\sqrt{2} \tan ^{-1}\left(\frac{x-1}{\sqrt{2 x}}\right), \\
\lim _{x \rightarrow 1} F_{1}(x)=0, \\
\lim _{x \rightarrow+\infty} F_{1}(x)=-\frac{\sqrt{2}}{2} \pi<0, \\
F_{1}^{\prime}(x)=-\frac{2(x-1)}{\sqrt{x}\left(x^{2}+1\right)\left(x^{p+1}+2 x^{p}-x^{p-1}-x^{2}+2 x+1\right)^{2}} f(x),
\end{gathered}
$$

where $f(x)$ is defined by (2.1).

We divide the proof into four cases.

Case 1. $p=2 \log 2 /(5 \log 2-2 \log \pi)$ Then it follows from Lemma 1(2) and (2.24) that there exists $\lambda \in(1,+\infty)$ such that $F_{1}(x)$ is strictly increasing on $(1, \lambda]$ and strictly decreasing on $[\lambda,+\infty)$.

Equations (2.21) and (2.22)-(2.23) together with the piecewise monotonicity of $F_{1}(x)$ lead to the conclusion that there exists $\lambda_{0} \in(1,+\infty)$ such that $F(x)$ is strictly increasing on $\left(1, \lambda_{0}\right]$ and strictly decreasing on $\left[\lambda_{0},+\infty\right)$.

Note that (2.20) becomes

$$
\lim _{x \rightarrow+\infty} F(x)=0,
$$


Therefore,

$$
N_{G Q}(a, b)>M_{2 \log 2 /(5 \log 2-2 \log \pi)}(a, b)
$$

for all $a, b>0$ with $a \neq b$ follows from (2.17)-(2.19) and (2.25) together with the piecewise monotonicity of $F(x)$.

Case 2. $p=4 / 3$ Then it follows from Lemma 1(1) and (2.24) that $F_{1}(x)$ is strictly decreasing on $(1,+\infty)$.

Therefore,

$$
N_{G Q}(a, b)<M_{4 / 3}(a, b)
$$

for all $a, b>0$ with $a \neq b$ follows from (2.17)-(2.19) and (2.21)-(2.22) together with the monotonicity of $F_{1}(x)$.

Case $3 . p>2 \log 2 /(5 \log 2-2 \log \pi)$ Then (2.20) leads to

$$
\lim _{x \rightarrow+\infty} F(x)<0,
$$

Equations (2.17)-(2.18) together with inequality (2.26) imply that there exists large enough $M_{0}>1$ such that

$$
N_{G Q}(a, b)<M_{p}(a, b)
$$

for all $a, b>0$ with $x \in\left(M_{0},+\infty\right)$.

Case $4 . p<4 / 3$ Let $x>0, x \rightarrow 0$, then making use the Taylor expansion we get

$$
\begin{aligned}
& N_{G Q}(1,1+x)-M_{p}(1,1+x) \\
& \begin{array}{r}
=\frac{2 x \sqrt{x+1}+\sqrt{2}\left[(x+1)^{2}+1\right] \tan ^{-1}\left(\frac{x}{\sqrt{2(x+1)}}\right)}{4 x}-\left[\frac{1+(1+x)^{p}}{2}\right]^{1 / p} \\
=\frac{4-3 p}{24} x^{2}+o\left(x^{2}\right) .
\end{array}
\end{aligned}
$$

Equation (2.27) implies that there exists small enough $\delta_{0}>0$ such that

$$
N_{G Q}(1,1+x)>M_{p}(1,1+x)
$$

for all $a, b>0$ with $x \in\left(0, \delta_{0}\right)$.

Therefore, Theorem 1 follows easily from Cases $1-4$ and the monotonicity of the function $p \rightarrow M_{p}(a, b)$.

\section{ACKNOWLEDGEMENT}

The research was supported by the Natural Science Foundation of China under Grants 61374086, 11171307 and 11401191, the Natural Science Foundation of Zhejiang Province under Grant LY13A010004, and the Natural Science Foundation of Zhejiang Broadcast and TV University under Grant XKT-17Z04 and XKT-17G26. 


\section{REFERENCES}

[1] Y.-M. Chu, W.-M. Qian, L.-M. Wu, and X.-H. Zhang, "Optimal bounds for the first and second Seiffert means in terms of geometric, arithmetic and contraharmonic means," J. Inequal. Appl., vol. 2015,44, pp. 1-9, 2015.

[2] Y.-M. Chu, S.-L. Qiu, and M.-K. Wang, "Sharp inequalities involving the power mean and complete elliptic integral of the first kind," Rocky Mountain J. Math., vol. 43, no. 5, pp. 1489-1496, 2013.

[3] Y.-M. Chu, Y.-F. Qiu, and M.-K. Wang, "Hölder means inequalities for the complete elliptic integrals," Integral Transforms Spec. Funct., vol. 23, no. 7, pp. 521-527, 2012.

[4] Y.-M. Chu, M.-K. Wang, Y.-P. Jiang, and S.-L. Qiu, "Concavity of the complete elliptic integrals of the second kind with respect to Hölder means," J. Math. Anal. Appl., vol. 395, no. 2, pp. 637-642, 2012.

[5] Y.-M. Chu and W.-F. Xia, "Two optimal double inequalities between power mean and logarithmic," Comput. Math. Appl., vol. 60, no. 1, pp. 83-89, 2010.

[6] Y.-M. Chu, Z.-H. Yang, and L.-M. Wu, "Sharp power mean bounds for Sándor mean,” Abstr. Appl. Anal., vol. ID172867, p. 5pages, 2015.

[7] I. Costin and G. Toader, "Optimal evaluations of some Seiffert-type means by power means," Appl. Math. Comput., vol. 219, no. 9, pp. 4745-4754, 2013.

[8] P. A. Hästö, "A monotonicity property of ratios of symmetric homogeneous means," J. Inequal. Pure appl. Math., vol. 3, no. 5, p. article 71, 2002.

[9] P. A. Hästö, "Optimal inequalities between Seiffert's mean and power means," Math. Inequal. Appl., vol. 7, no. 1, pp. 47-53, 2004.

[10] A. A. Jagers, "Solution of problem 887," Nieuw Arch. Wisk., vol. 12, no. 2, pp. 230-231, 1994.

[11] J.-F. Li, Z.-H. Yang, and Y.-M. Chu, "Optimal power mean bounds for the second Yang mean," $J$. Inequal. Appl., vol. Article 31, p. 9pages, 2016.

[12] Y.-M. Li, M.-K. Wang, and Y.-M. Chu, "Sharp power mean bounds for Seiffert mean," Appl. Math. J. Chinese Univ., vol. 29B, no. 1, pp. 101-107, 2014.

[13] T. P. Lin, "The power mean and the logarithmic mean," Amer. Math. Monthly, vol. 81, no. 8, pp. 337-343, 1974.

[14] P. B. D. Mitrinović and P. M. Vasić, Means and Their Inequalities. Dordrecht, 1988.

[15] E. Neuman, "On a new bivariate mean," Aequat. Math., vol. 88, no. 3, pp. 277-289, 2014.

[16] E. Neuman and J. Sándor, "On the Schwab-Borchardt mean," Mathematica Pannonica, vol. 14, no. 2, pp. 253-266, 2003.

[17] E. Neuman and J. Sándor, "On the Schwab-Borchardt mean II," Mathematica Pannonica, vol. 17, no. 1, pp. 49-59, 2006.

[18] A. O. Pittenger, "Inequalities between arithmetic and logarithmic means," Univ. Beograd. Publ. Elektrotehn. Fak. Ser. Math. Fiz., vol. 1980, no. 678-715, pp. 15-18, 1980.

[19] W.-M. Qian, Z.-H. Shao, and Y.-M. Chu, "Sharp inequalities involving Neuman means of the second kind," J. Math. Inequal., vol. 9, no. 2, pp. 531-540, 2015.

[20] T. Radó, "On convex functions," Trans. Amer. Math. Soc., vol. 37, no. 2, pp. 266-285, 1935.

[21] L.-C. Shen, Y.-Y. Yang, and W.-M. Qian, "Sharp inequalities involving Neuman means of the second kind with applications," Journal of Advances in Applied Mathematics, vol. 1, no. 3, pp. 139-148, 2016.

[22] Y.-Q. Song, T.-H. Zhao, Y.-M. Chu, and X.-H. Zhang, "Optimal evaluation of a Toader-type mean by power mean," J. Inequal. Appl., vol. Article 408, p. 12pages, 2015.

[23] K. B. Stolarsky, "The power and generalized logarithmic means," Amer. Math. Monthly, vol. 87, no. 7, pp. 545-548, 1980. 
[24] G.-D. Wang, X.-H. Zhang, and Y.-M. Chu, "A power mean inequality involving the complete elliptic integrals," Rocky Mountain J. Math., vol. 44, no. 5, pp. 1661-1667, 2014.

[25] M.-K. Wang and Y.-M. Chu, "Refinements of transformation inequalities for zero-balanced hypergeometric functions," Acta Math. Sci., vol. 37B, no. 3, pp. 607-622, 2017.

[26] M.-K. Wang, Y.-M. Chu, Y.-F. Qiu, and S.-L. Qiu, “An optimal power mean inequality for the complete elliptic integrals," Appl. Math. Lett., vol. 24, no. 6, pp. 887-890, 2011.

[27] M.-K. Wang, Y.-M. Li, and Y.-M. Chu, "Inequalities and infinite product formula for Ramanujian generalized modular equation function,” Ramanujan. J., pp. 1-12, 2017, doi: 10.1007/s11139017-9888-3.

[28] Z.-H. Yang, "Estimates for Neuman-Sándor mean by power means and their relative errors," $J$. Math. Inequal., vol. 7, no. 4, pp. 711-726, 2013.

[29] Z.-H. Yang, "Three families of two-parameter means constructed by trigonometric functions," $J$. Inequal. Appl., vol. 2013,541, pp. 1-27, 2013.

[30] Z.-H. Yang, L.-M. Wu, and Y.-M. Chu, “Optimal power mean bounds for Yang mean,” J. Inequal. Appl., vol. 2014,401, pp. 1-10, 2014.

[31] Z.-H. Yang and Y.-M. Chu, "Optimal evaluations for the Sándor-Yang mean by power mean," Math. Inequal. Appl., vol. 19, no. 3, pp. 1031-1038, 2016.

[32] Z.-H. Yang, Y.-M. Chu, and M.-K. Wang, "Monotonicity criterion for the quotient of power series with applications," J. Math. Anal. Appl., vol. 428, no. 1, pp. 587-604, 2015.

[33] Z.-H. Yang, Y.-M. Chu, and W. Zhang, "Accurate approximations for the complete elliptic integal of the second kind," J. Math. Anal. Appl., vol. 438, no. 2, pp. 875-888, 2016.

[34] Z.-H. Yang, Y.-M. Chu, and W. Zhang, "Monotonicity of the ratio for the complete elliptic integral and Stolarsky mean," J. Inequal. Appl., vol. 1016, no. 176, p. 10pages, 2016.

[35] Z.-H. Yang, Y.-M. Chu, and X.-H. Zhang, "Sharp Stolarsky mean bounds for the complete elliptic integral of the second kind," J. Nonlinear Sci. Appl., vol. 10, no. 3, pp. 929-936, 2017.

[36] X.-H. Zhang, G.-D. Wang, and Y.-M. Chu, "Convexity with respect to Hölder mean involving zero-balanced hypergeometric functions," J. Math. Anal. Appl., vol. 353, no. 1, pp. 256-259, 2009.

Authors' addresses

Xiao-Hong He

Office of Academic Affairs, Quzhou Broadcast and TV University, Quzhou, ZheJiang, China

E-mail address: mcqmm20060163.com

Yue-Ying Yang

School of Mechanical and Electrical Engineering, Huzhou Vocational \& Technical College, Huzhou, ZheJiang, China

E-mail address: yyy1008hz@163.com

Wei-Mao Qian

School of Distance Education, Huzhou Broadcast and TV University, Huzhou, ZheJiang, China

E-mail address: qwm661977@126.com 